\title{
Surface potential measurements by the dissipative force modulation method
}

\section{$\operatorname{AUTHOR}(\mathrm{S}):$}

Fukuma, Takeshi; Kobayashi, Kei; Yamada, Hirofumi; Matsushige, Kazumi

\section{CITATION:}

Fukuma, Takeshi ... [et al]. Surface potential measurements by the dissipative force modulation method. REVIEW OF SCIENTIFIC INSTRUMENTS 2004, 75(11): 4589-4594

\section{ISSUE DATE:}

2004-11

URL:

http://hdl.handle.net/2433/39810

\section{RIGHT:}

Copyright 2004 American Institute of Physics. This article may be downloaded for personal use only. Any other use requires prior permission of the author and the American Institute of Physics. 


\title{
Surface potential measurements by the dissipative force modulation method
}

\author{
Takeshi Fukuma \\ Department of Electronic Science and Engineering, Kyoto University, Kyoto 606-8501, Japan \\ Kei Kobayashi \\ International Innovation Center, Kyoto University, Kyoto 606-8501, Japan \\ Hirofumi Yamada ${ }^{\text {a) }}$ and Kazumi Matsushige \\ Department of Electronic Science and Engineering, Kyoto University, Kyoto 606-8501, Japan
}

(Received 21 June 2004; accepted 20 August 2004; published 29 October 2004)

\begin{abstract}
In this study, we propose a novel surface property measurement technique using noncontact atomic force microscopy (NC-AFM), which is referred to as the "dissipative force modulation (DM) method." NC-AFM-based surface property measurements have mostly utilized conservative tip-sample interaction forces, which induce a frequency shift of cantilever resonance without dissipating cantilever vibration energy. In the DM method, local surface properties are measured by detecting a modulated dissipative tip-sample interaction force which dissipates cantilever vibration energy and hence induces an amplitude variation in cantilever vibration. Since the force sensitivity to dissipative interactions obtained in a typical NC-AFM setup is much higher than that to conservative ones, the DM method can improve the sensitivities of conventional NC-AFM-based techniques that utilize conservative interactions. Combining this method with Kelvin-probe force microscopy, we present the first quantitative surface potential measurement through dissipative tip-sample interactions. (c) 2004 American Institute of Physics. [DOI: 10.1063/1.1805291]
\end{abstract}

\section{INTRODUCTION}

Noncontact atomic force microscopy (NC-AFM) using the frequency modulation (FM) detection method ${ }^{1}$ has attracted much attention due to its capability of imaging atomic-scale structures even on insulating surfaces ${ }^{2}$ as well as on conductive surfaces. ${ }^{3,4}$ In addition to the imaging of surface structures, NC-AFM has also been used for the investigation of local surface properties at a nanometer-scale resolution. In particular, Kelvin-probe force microscopy (KFM) combined with NC-AFM (Ref. 5) has been applied to the measurement of local surface potential distributions at a nearly atomic-scale resolution.

In NC-AFM, a microfabricated cantilever with a sharp tip mounted at its end is brought close to the surface to detect various tip-sample interaction forces. These tip-sample interaction forces detected in NC-AFM are classified into two categories: "conservative" forces and "dissipative" forces. ${ }^{6,7}$ Conservative forces induce a frequency shift of cantilever resonance without dissipating cantilever vibration energy. On the other hand, dissipative forces reduce cantilever vibration amplitude, which means that the mechanical energy of the cantilever is dissipated through some of the tip-sample interactions.

Since tip-sample interaction forces in NC-AFM are mostly conservative, conservative forces, rather than dissipative forces, have been utilized thus far in NC-AFM applications. The frequency shift induced by a conservative tip-

\footnotetext{
${ }^{\text {a)} E l e c t r o n i c ~ m a i l: ~ h-y a m a d a @ k u e e . k y o t o-u . a c . j p ~}$
}

sample interaction force is detected and used for tip-sample distance regulation. In KFM, an ac bias voltage is applied between a tip and a sample, which modulates the magnitude of a conservative electrostatic force. Then the resultant change in cantilever resonance frequency is detected and used for bias feedback regulation. ${ }^{5}$

In contrast to the conservative force measurements, surface property measurements hardly use dissipative forces. This is because energy dissipation in NC-AFM has different origins related to the electrical and mechanical properties of a tip and a sample. ${ }^{8,9}$ Thus, the quantitative evaluation of surface properties is difficult by a simple measurement of the total amount of energy dissipation. However, previously reported energy dissipation values measured by NC-AFM have suggested that the force sensitivity to dissipative interactions obtained with a typical NC-AFM setup is much higher than that to conservative ones. ${ }^{10,11}$ For example, an energy dissipation of less than $1 \mathrm{fW}$ was accurately measured in previous studies, ${ }^{10,11}$ which means that a dissipative electrostatic force of less than $0.01 \mathrm{pN}$ is readily detected in NC-AFM. This indicates that the use of dissipative forces instead of conservative ones should improve such sensitivity in surface property measurements.

In this article, we propose a novel NC-AFM-based technique referred to as the "dissipative force modulation (DM) method." By introducing and detecting a modulated dissipative force, the method enables the separation of the dissipative interaction of interest. Combining this method with KFM, we have developed a modified type of KFM that enables quantitative surface potential measurement with an ex- 
tremely high sensitivity. To show the clear contrast between the currently used KFM and the newly developed one, we hereafter describe these two methods as conservative force modulation KFM (CM-KFM) and dissipative force modulation KFM (DM-KFM), respectively. In this article, the basic principle and experimental setup of DM-KFM are presented. The extremely high sensitivity of NC-AFM to a dissipative interaction force is experimentally demonstrated. In addition, the preliminary results of surface potential measurements using DM-KFM are presented.

\section{BASIC PRINCIPLE}

\section{A. Conservative and dissipative forces}

In NC-AFM, the phase difference between cantilever oscillation and its excitation signal $\left(v_{\text {exc }}\right)$ is continuously kept constant at $90^{\circ}$ with a self-excitation circuit. Thus, $v_{\text {exc }}$ and tip position $\left(z_{t}\right)$ can be described as

$$
\begin{aligned}
& v_{\text {exc }}=V_{\text {exc }} \cos (\omega t), \\
& z_{t}=z_{t 0}+A \sin (\omega t) .
\end{aligned}
$$

$V_{\text {exc }}$ and $\omega$ are the amplitude and frequency of the cantilever excitation signal, respectively. $z_{t 0}$ and $A$ denote the mean tip position and the amplitude of the cantilever vibration, respectively.

Due to the high $Q$-factor of the cantilever, cantilever motion, particularly in vacuum, is predominantly affected by the $\omega$-components of tip-sample interaction forces. Accordingly, the tip-sample interaction force $\left(F_{t s}\right)$ can be approximately described by two trigonometric functions whose phases differ by $90^{\circ}$,

$$
F_{t s}=F_{t s c} \sin (\omega t)+F_{t s d} \cos (\omega t) .
$$

The first component $\left(F_{t s c} \sin (\omega t)\right)$ changes with the same phase as that of the cantilever vibration, which induces a frequency shift $(\Delta f)$ of cantilever resonance without dissipating vibration energy. On the other hand, the second component $\left(F_{t s d} \cos (\omega t)\right)$ changes with the same phase as that of the cantilever excitation signal, which dissipates some energy of the cantilever vibration. The energy dissipation results in an amplitude variation $(\Delta A)$ of the cantilever oscillation. In this article, we refer to the former component as conservative force and to the latter as dissipative force.

From the equation of motion, $\Delta f$ and $\Delta A$ are given by

$$
\begin{aligned}
& \Delta f=-\frac{f_{0}}{2 k A} F_{t s c}, \\
& \Delta A=\frac{Q}{k} F_{t s d},
\end{aligned}
$$

where $f_{0}, k$, and $Q$ are the resonance frequency, the spring constant and the $Q$-factor of the cantilever, respectively. Thus, the minimum detectable force for conservative interactions $\left(\delta F_{t s c}\right)$ and that for dissipative interactions $\left(\delta F_{t s d}\right)$ are given by

$$
\delta F_{t s c}=\frac{2 k A}{f_{0}} \delta f,
$$

TABLE I. Typical values of parameters of cantilever under vacuum and experimental conditions in NC-AFM experiments.

\begin{tabular}{ccc}
\hline \hline Parameter & Value & Unit \\
\hline$f_{0}$ & 300 & $\mathrm{kHz}$ \\
$k$ & 40 & $\mathrm{~N} / \mathrm{m}$ \\
$Q$ & 30000 & \\
$T$ & 300 & $\mathrm{~K}$ \\
$f_{m}$ & 1 & $\mathrm{kHz}$ \\
$B$ & 200 & $\mathrm{~Hz}$ \\
$n_{\mathrm{ds}}$ & $0.1-1$ & $\mathrm{pm} / \sqrt{\mathrm{Hz}}$ \\
$A$ & 5 & $\mathrm{~nm}$ \\
$z_{t 0}$ & 6 & $\mathrm{~nm}$ \\
$R$ & 5 & $\mathrm{~nm}$ \\
\hline \hline
\end{tabular}

$$
\delta F_{t s d}=\frac{k}{Q} \delta A
$$

where $\delta f$ and $\delta A$ are the minimum detectable frequency and amplitude, respectively.

There are two major noise sources that limit the sensitivities to frequency and amplitude in NC-AFM, which are the thermal vibration of the cantilever and noise from the deflection sensor. In both CM- and DM-KFM, a conservative force or a dissipative electrostatic force is modulated at a frequency of $f_{m}$ by applying an ac bias voltage $\left(f_{m} \ll f_{0}\right)$. Thus, the spectral noise density of a cantilever deflection signal at a frequency of $f_{0}+f_{m}$ has to be taken into account for the evaluation of the force sensitivities. For the noise arisig from the cantilever thermal vibration, the root-meansquare (RMS) value of spectral noise density $\left(n_{\mathrm{th}}\right)$ at a frequency of $f_{0}+f_{m}$ is approximately expressed by ${ }^{1}$

$$
n_{\mathrm{th}}=\sqrt{\frac{k_{B} T f_{0}}{2 \pi k Q f_{m}^{2}}} .
$$

Table I shows an example of typical cantilever parameters under vacuum and experimental conditions. Under these conditions, $n_{\text {th }}$ is $13 \mathrm{fm} / \sqrt{\mathrm{Hz}}$. On the other hand, the typical RMS value of the spectral noise density arising from a deflection sensor $\left(n_{\mathrm{ds}}\right)$ falls in the range of $0.1-1 \mathrm{pm} / \sqrt{\mathrm{Hz}}$, which is much larger than $n_{\mathrm{th}}$. Thus, $n_{\mathrm{ds}}$ predominantly determines $\delta f$ and $\delta A$ for typical NC-AFM setups operating in vacuum.

Assuming that the modulated frequency and amplitude are detected with a lock-in amplifier with a bandwidth of $B$, $\delta f$, and $\delta A$ at a modulation frequency of $f_{m}$ are, respectively, given by ${ }^{12}$

$$
\begin{aligned}
& \delta f=\frac{\sqrt{12}}{\pi A} f_{m} n_{\mathrm{ds}} \sqrt{B}, \\
& \delta A=n_{\mathrm{ds}} \sqrt{B} .
\end{aligned}
$$

Note that the condition $f_{m}^{2} \gg B^{2}$ is assumed in obtaining $\delta f$. With the typical conditions given in Table I, $\delta f$ is approximately $0.3-3 \mathrm{~Hz}$ while $\delta A$ is approximately $1.4-14 \mathrm{pm}$.

From Eqs. (6), (7), (9), and (10), $\delta F_{t s c}$ and $\delta F_{t s d}$ are, respectively, obtained as 


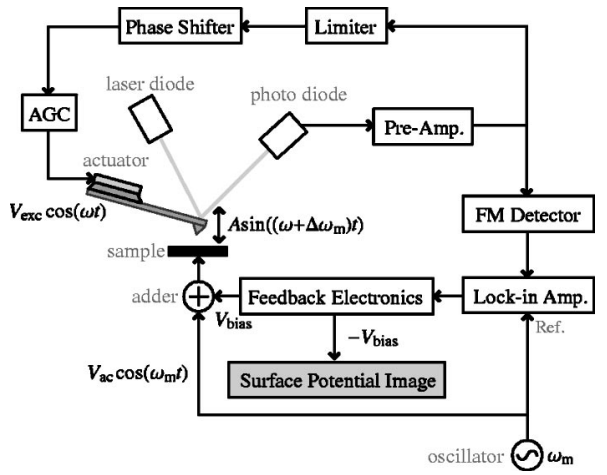

FIG. 1. Schematic of experimental setup for CM-KFM. The frequency variation induced by the modulated conservative electrostatic force is detected for obtaining $V_{\mathrm{CPD}}$.

$$
\begin{aligned}
& \delta F_{t s c}=\frac{4 \sqrt{3} k}{\pi} \frac{f_{m}}{f_{0}} n_{\mathrm{ds}} \sqrt{B}, \\
& \delta F_{t s d}=\frac{k}{Q} n_{\mathrm{ds}} \sqrt{B} .
\end{aligned}
$$

Under the typical conditions shown in Table I, $\delta F_{t s c}$ is approximately $0.4-4 \mathrm{pN}$ while $\delta F_{t s d}$ is approximately 2 $-20 \mathrm{fN}$. Therefore, owing to the high $Q$-factor of the cantilever in vacuum, the force sensitivity to dissipative interactions obtained with a typical NC-AFM setup is much higher than that to conservative ones.

\section{B. Conservative force modulation method}

When a dc bias voltage is applied between a tip and a sample, the induced electrostatic force $\left(F_{\text {es }}\right)$ changes with the same frequency and phase as those of the cantilever vibration. This is because $F_{\text {es }}$ is a function of tip position which changes according to Eq. (2). Thus, the induced electrostatic force is naturally conservative. In CM-KFM, a bias voltage oscillating with a frequency $\left(\omega_{m}\right)$ much lower than that of the cantilever vibration is applied between the tip and the sample for introducing a modulated conservative electrostatic force.

Figure 1 shows an experimental setup for CM-KFM. In CM-KFM, an ac bias voltage $V_{\text {ac }} \cos \left(\omega_{m} t\right)$ and a dc bias voltage $V_{\text {bias }}$ is applied between a tip and a sample. Accordingly, the tip-sample potential difference $V_{t s}$ is given by

$$
V_{t s}=V_{\mathrm{dc}}+V_{\mathrm{ac}} \cos \left(\omega_{m} t\right)
$$

where

$$
V_{\mathrm{dc}}=V_{\mathrm{bias}}+V_{\mathrm{CPD}} .
$$

Here $V_{\mathrm{CPD}}$ is the contact potential difference between the tip and the sample and $V_{\mathrm{dc}}$ is defined as the sum of $V_{\text {bias }}$ and $V_{\mathrm{CPD}}$.

$$
\begin{aligned}
& F_{\text {es }} \text { induced by } V_{t s} \text { is given by }{ }^{13} \\
& F_{\text {es }}=-\frac{\pi \varepsilon_{0} R}{z_{t}} V_{t s}^{2},
\end{aligned}
$$

where $\varepsilon_{0}$ and $R$ are the dielectric constant in vacuum and the tip radius, respectively. Using Eq. (2), the equation is rewritten as

$$
F_{\mathrm{es}}=-\frac{\pi \varepsilon_{0} R}{z_{t 0}}\left[1-\frac{A}{z_{t 0}} \sin (\omega t)\right] V_{t s}^{2},
$$

where we assume that $A \ll z_{t 0}$. Although this assumption is not always satisfied in conventional NC-AFM, we have checked that no significant difference was made in the following discussion even without this assumption. Thus, we still keep it to have the essential understanding by simple calculation. From Eq. (16), the conservative electrostatic force $\left(F_{\text {esc }}\right)$ is given by

$$
F_{\mathrm{esc}}=\frac{\pi \varepsilon_{0} R A}{z_{t 0}^{2}} V_{t s}^{2},
$$

while the dissipative electrostatic force $\left(F_{\text {esd }}\right)$ is zero.

From Eqs. (4), (13), and (17), the $\omega_{m}$ component of the frequency shift $\left(\Delta f_{m}\right)$ induced by the electrostatic interaction is given by

$$
\Delta f_{m}=-\frac{\pi \varepsilon_{0} R f_{0} V_{\mathrm{ac}}}{k z_{t 0}^{2}} V_{\mathrm{dc}} \cos \left(\omega_{m} t\right) .
$$

This frequency variation is detected with a lock-in amplifier from the output signal of a frequency modulation (FM) detector. Then the detected signal is fed into the feedback electronics that controls $V_{\text {bias }}$ so as to make $V_{\mathrm{dc}}$ zero. Consequently, the surface potential image is obtained by recording $-V_{\text {bias }}$ as the tip is scanning over a surface.

From Eqs. (9) and (18), the minimum detectable contact potential difference $\delta V_{\mathrm{CPD}}$ is given by

$$
\delta V_{\mathrm{CPD}}=\frac{2 \sqrt{6}}{\pi^{2} \varepsilon_{0}} \frac{k z_{t 0}^{2}}{R A V_{\mathrm{ac}}} \frac{f_{m}}{f_{0}} n_{\mathrm{ds}} \sqrt{B} .
$$

Using the typical conditions given in Table I, $\delta V_{\mathrm{CPD}}$ is approximately $15-150 \mathrm{mV}$ at $V_{\mathrm{ac}}$ of $1 \mathrm{~V}$. In other words, to obtain a potential resolution higher than $10 \mathrm{mV}, V_{\mathrm{ac}}$ has to be higher than $1 \mathrm{~V}$.

The application of an ac bias voltage also produces a dc component of the frequency shift $\left(\Delta f_{\mathrm{dc}}\right)$ as well as the $\omega_{\mathrm{m}}$ component. This dc component remains even when the bias feedback regulation reaches a steady state $\left(V_{\mathrm{dc}}=0\right)$ and is given by

$$
\Delta f_{\mathrm{dc}}=-\frac{\pi \varepsilon_{0} R f_{0}}{4 k z_{t 0}^{2}} V_{\mathrm{ac}}^{2} .
$$

Under the typical conditions shown in Table I, $\Delta f_{\mathrm{dc}}$ is $7.2 \mathrm{~Hz}$ at $\mathrm{V}_{\mathrm{ac}}=1 \mathrm{~V}$. This value is not negligible compared with the typical frequency shift values used for topographic imaging, which ranges from 10 to $100 \mathrm{~Hz}$. Thus, $\Delta f_{\text {dc }}$ can cause topographic artifacts as previously reported. ${ }^{14}$ In particular, for KFM applications to insulating thin films, such as organic thin films, on metal surfaces, $z_{t 0}$ should be defined as the distance between the metal surface and the tip position. In addition, the dielectric constant between the tip and the metal surface can vary depending on the type of film material. Accordingly, site-dependent variations in $z_{t 0}$ and dielectric constant can result in topographic artifacts due to the variation in $\Delta f_{\mathrm{dc}}$. This is one of the major problems in CM-KFM. 


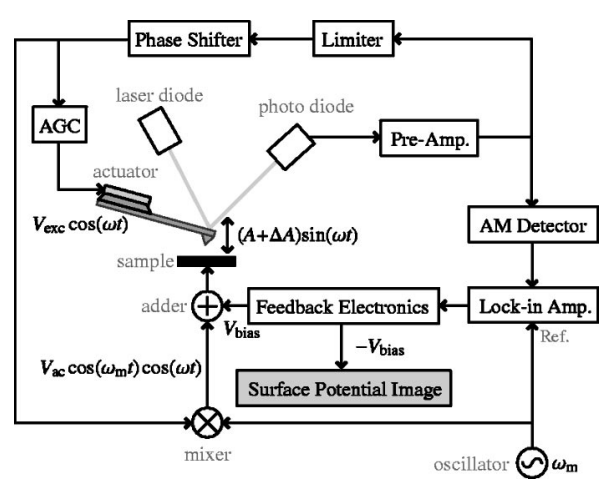

FIG. 2. Schematic of experimental setup for DM-KFM, which can be obtained with only small modifications of that for CM-KFM. The amplitude variation induced by the modulated dissipative electrostatic force is detected for obtaining $V_{\mathrm{CPD}}$.

\section{Dissipative force modulation method}

Since the electrostatic force in a simple CM-KFM setup is conservative, we need to modify the setup to detect $F_{\text {es }}$ as the dissipative force $\left(F_{\text {esd }}\right)$. The required dissipative force must have the same frequency as that of the cantilever oscillation but a $90^{\circ}$ phase difference. The cantilever excitation signal in a self-excitation circuit which coincides with these conditions can be an appropriate candidate for generating the dissipative force. In DM-KFM, $F_{\text {esd }}$ is introduced by applying an ac bias voltage synchronized with the cantilever excitation signal. Then the amplitude of the ac bias voltage is modulated at a frequency $\left(\omega_{m}\right)$ much lower than that of the cantilever vibration $(\omega)$, producing a modulated dissipative electrostatic force.

Figure 2 shows an experimental setup for DM-KFM. Note that the tip-sample distance is regulated in the constant frequency shift mode although this part of the setup is omitted in Fig. 2 to avoid complication. In DM-KFM, an ac bias voltage $V_{\text {ac }} \cos \left(\omega_{m} t\right) \cos (\omega t)$ and a dc bias voltage $V_{\text {bias }}$ are applied between the tip and the sample. The resultant tipsample potential difference $V_{t s}$ is given by

$$
V_{t s}=V_{\mathrm{dc}}+V_{\mathrm{ac}} \cos \left(\omega_{m} t\right) \cos (\omega t) .
$$

Note that $V_{\mathrm{dc}}$ has been defined in Eq. (14). From Eqs. (14), (16), and (21), the electrostatic force $\left(F_{\text {es }}\right)$ produced by the application of the bias voltage is given by

$$
\begin{aligned}
F_{\mathrm{es}}= & \frac{\pi \varepsilon_{0} R A}{z_{\mathrm{t} 0}^{2}}\left[V_{\mathrm{dc}}^{2}+\frac{1}{8} V_{\mathrm{ac}}^{2}\left\{1+\cos \left(2 \omega_{m} t\right)\right\}\right] \sin (\omega t) \\
& -\frac{2 \pi \varepsilon_{0} R}{z_{t 0}} V_{\mathrm{ac}} V_{\mathrm{dc}} \cos \left(\omega_{m} t\right) \cos (\omega t),
\end{aligned}
$$

where we take the components with the first order of $\sin (\omega t)$ or $\cos (\omega t)$ into account.

The second term on the right-hand side of Eq. (22) corresponds to the dissipative electrostatic force which induces amplitude variation $(\Delta A)$ described by

$$
\Delta A=-\frac{2 \pi \varepsilon_{0} R}{z_{t 0}} \frac{Q}{k} V_{\mathrm{ac}} V_{\mathrm{dc}} \cos \left(\omega_{m} t\right) .
$$

The amplitude variation is detected with a lock-in amplifier from the output signal of an amplitude modulation (AM) detector with a bandwidth larger than $\omega_{m}$. The detected sig- nal is fed into the feedback electronics that control $V_{\text {bias }}$ for canceling out the $\omega_{m}$ component of amplitude variation. Consequently, $V_{\text {bias }}$ is kept equal to $-V_{\mathrm{CPD}}$. Thus, a surface potential image can be obtained by two-dimensionally mapping the values of $-V_{\text {bias }}$.

From Eqs. (10) and (23), the minimum detectable contact potential difference $\left(\delta V_{\mathrm{CPD}}\right)$ is given by

$$
\delta V_{\mathrm{CPD}}=\frac{1}{\sqrt{2} \pi \varepsilon_{0}} \frac{k z_{t 0}}{R Q V_{\mathrm{ac}}} n_{d s} \sqrt{B} .
$$

Under the typical conditions shown in Table $\mathrm{I}, \delta V_{\mathrm{CPD}}$ is $0.58-5.8 \mathrm{mV}$ at a $V_{\mathrm{ac}}$ of $0.1 \mathrm{~V}$. That is, $V_{\mathrm{ac}}$ of $0.1 \mathrm{~V}$ is sufficiently high for obtaining a potential resolution of 10 $\mathrm{mV}$. Owing to the high force sensitivity of DM-KFM, we can achieve a sufficient potential resolution with smaller $V_{\text {ac }}$ values than those required for CM-KFM.

The first term on the right-hand side of Eq. (22) represents the conservative electrostatic force that causes a frequency shift of the cantilever resonance. The dc frequency shift $\left(\Delta f_{\mathrm{dc}}\right)$ in a steady state under the bias feedback control $\left(V_{\text {bias }}=-V_{\mathrm{CPD}}\right)$ is given by

$$
\Delta f_{\mathrm{dc}}=-\frac{\pi \varepsilon_{0} R f_{0}}{16 k z_{t 0}^{2}} V_{\mathrm{ac}}^{2} .
$$

Comparing this equation with Eq. (20), one can find that $\Delta f_{\mathrm{dc}}$ in DM-KFM is $1 / 4$ that in CM-KFM. In addition, $\Delta f_{\mathrm{dc}}$ can be decreased to a negligible value because small $V_{\text {ac }}$ values are available in DM-KFM. For example, under the typical conditions shown in Table I, $\Delta f_{\mathrm{dc}}$ is $0.018 \mathrm{~Hz}$ at $V_{\mathrm{ac}}$ of $0.1 \mathrm{~V}$. The result shows that DM-KFM enables high resolution potential measurements without inducing topographic artifacts. The reduction in $V_{\mathrm{ac}}$ is also beneficial for suppressing the influence of bias voltage on sample properties to be measured by KFM.

\section{RESULTS AND DISCUSSIONS}

\section{A. Signal-to-noise ratio measurements}

Although the previously reported energy dissipation values obtained with NC-AFM have suggested that the force sensitivity of NC-AFM to dissipative interactions is higher than that to conservative ones, ${ }^{10,11}$ quantitative comparison between these two force sensitivities has not yet been performed. In this study, we even compared the signal-to-noise ratio (SNR) of FM- and AM-detected signals generated by conservative and dissipative electrostatic forces at different bias modulation frequencies.

For the conservative force measurement, an ac bias voltage of $V_{\mathrm{ac}} \cos \left(\omega_{m} t\right)$ was applied between the tip and the sample and the modulated frequency shift was detected with an FM detector. On the other hand, for the dissipative force measurement, an ac bias voltage of $V_{\mathrm{ac}} \cos \left(\omega_{m} t\right) \cos (\omega t)$ was applied and the induced amplitude variation was detected with an AM detector. The bandwidths of the FM and AM detectors were $1 \mathrm{kHz}$. A commercially available ultrahigh vacuum (UHV) NC-AFM (JEOL: JSPM-4500) was used. A homebuilt PLL circuit using a voltage-controlled crystal oscillator (VCXO) (Ref. 15) was used for FM detection while an RMS-DC converter was used for AM detection. SNR was 

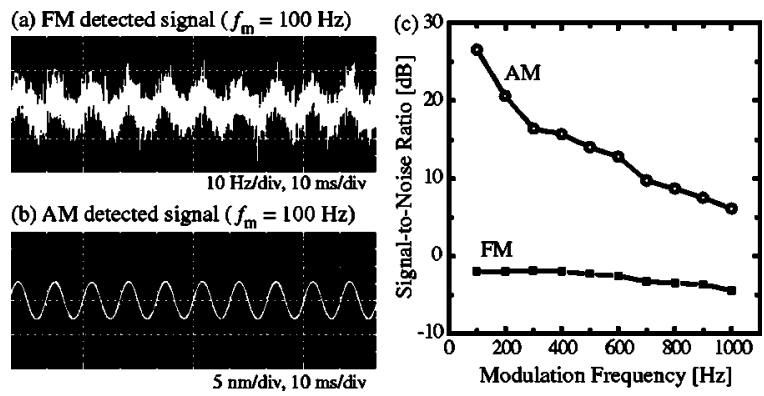

FIG. 3. (a), (b) Waveforms of FM- and AM-detected signals at modulation frequency of $100 \mathrm{~Hz}$. (c) SNRs of FM- and AM-detected signals plotted as functions of modulation frequency $\left(V_{\mathrm{ac}}=0.1 \mathrm{~V}, V_{\text {bias }}=1.0 \mathrm{~V}, A=5 \mathrm{~nm}, \Delta f\right.$ $=-20 \mathrm{~Hz}$ ).

measured with an FFT analyzer (softDSP: SDS-200). The cantilever was a Pt-coated $\mathrm{Si}$ cantilever (Nanosensors: NCHPt) with a nominal spring constant of $40 \mathrm{~N} / \mathrm{m}$ and a resonance frequency of approximately $300 \mathrm{kHz}$. The $Q$-factor measured under UHV conditions was approximately 30000 . The sample was a Pt thin film deposited on a $\mathrm{SiO}_{2} / \mathrm{Si}$ substrate. The measurements were performed at a tip position where $\Delta f=-20 \mathrm{~Hz}$. $V_{\text {bias }}$ and $V_{\text {ac }}$ were set at $1.0 \mathrm{~V}$ and $0.1 \mathrm{~V}$, respectively.

Figures 3(a) and 3(b) show the waveforms of FM- and AM-detected signals obtained at a modulation frequency of $100 \mathrm{~Hz}$, respectively. These waveforms reveal that the AMdetected signal has a much higher SNR than the FM-detected signal. This result experimentally demonstrates that the force sensitivity of NC-AFM to dissipative interactions is much higher than that to conservative ones. Figure 3(c) shows the frequency dependences of the SNRs of AM- and FMdetected signals. The result indicates that the SNR of the AM-detected signal decreases with increasing modulation frequency while the SNR of the FM-detected signal remains almost constant. However, the result also shows that the AMdetected signal still exhibits a higher SNR than the FMdetected signal even at a modulation frequency of $1 \mathrm{kHz}$.

The amplitude variation induced by a dissipative force settles on a time scale of $\tau_{\mathrm{AM}} \approx 2 Q / f_{0}$ while the response time for the frequency variation induced by a conservative force is given by $\tau_{\mathrm{FM}} \approx 1 / f_{0}{ }^{12}$ Namely, AM detection has a slower time response by a factor of $Q$ than that of FM detection, which decreases force sensitivity to dissipative forces at higher modulation frequencies. Thus, the DM method is most effective for applications that require an extremely high force sensitivity but not a very high scanning speed. The use of a high-resonance-frequency cantilever is the most effective way of enhancing force sensitivity and improving the time response of AM detection.

\section{B. Surface potential imaging}

Using DM- and CM-KFM, we have measured the surface potential distribution of a dimethylquinquethiophene (M5T) monolayer formed on a Pt surface. M5T molecules [Fig. 4(a)] deposited on a Pt surface form monolayer islands with their molecular axes perpendicular to the surface, as shown in Fig. 4(b). It has been reported that these monolayer (a) Dimethylquinquethiophene (M5T)

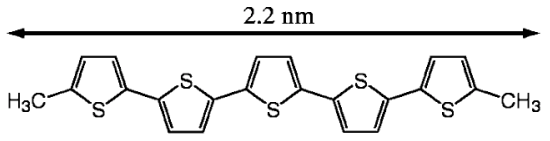

(b) M5T/Pt

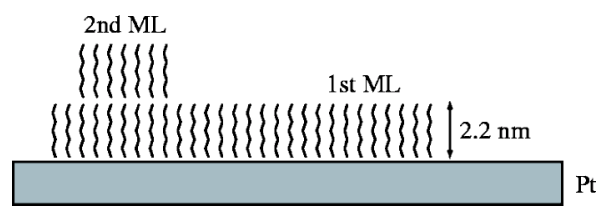

FIG. 4. (Color online) (a) Molecular structure of the M5T molecule. (b) Schematic model of the M5T monolayer formed on a Pt surface.

islands have 100-200 $\mathrm{mV}$ higher surface potential than $\mathrm{Pt}$ surfaces. $^{16}$

Figure 5 shows the topographic and potential images taken by CM- and DM-KFM. The film/substrate potential difference measured from these two potential images [(Figs. 5(b) and 5(d)] agreed well and the value was approximately $100 \mathrm{mV}$. Owing to the excellent SNR of AM detection, the potential image obtained by DM-KFM shows a much clearer contrast than the CM-KFM image. Namely, the result demonstrates that DM-KFM has a higher potential resolution than CM-KFM.

In CM-KFM, it was difficult to obtain a clear surface potential image with $V_{\text {ac }}$ values of less than approximately $1 \mathrm{~V}$ while clear potential contrast was obtained in DM-KFM even with $V_{\mathrm{ac}}$ of $0.1 \mathrm{~V}$ as shown in Fig. 5(d). The result shows that DM-KFM enables us to achieve a sufficiently high potential sensitivity even with a small $V_{\text {ac }}$, markedly suppressing the possible formation of topographic artifacts and the influence of the bias application on the sample properties. DM-KFM is also suitable for NC-AFM operation with a small cantilever vibration amplitude, which has been recently proven to be beneficial for enhancing spatial resolution in topographic imaging. ${ }^{17} \delta V_{\mathrm{CPD}}$ for CM-KFM increases
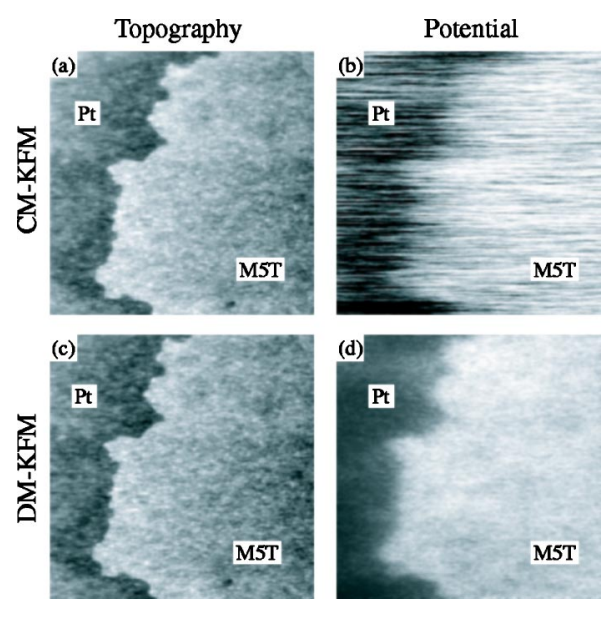

FIG. 5. (Color online) NC-AFM images of the M5T monolayer on a Pt surface. (a) Topographic and (b) potential images obtained by CM-KFM. (c) Topographic and (d) potential images obtained by DM-KFM. The experimental parameters used in both CM- and DM-KFM: $\Delta f=-20 \mathrm{~Hz}, A$ $=1 \mathrm{~nm}, V_{\mathrm{ac}}=0.1 \mathrm{~V}, f_{m}=1 \mathrm{kHz}$. The scanned area and imaging speed were $1 \mu \mathrm{m} \times 1 \mu \mathrm{m}$ and $15 \mathrm{~min} /$ frame, respectively. 
with decreasing cantilever vibration amplitude while that of DM-KFM remains almost constant as expected from Eqs. (19) and (24).

In terms of the spatial resolution of a potential image, CM-KFM has an advantage over DM-KFM. Comparing Eqs. (18) and (23), we can find that $\Delta f_{m}$ is proportional to $1 / z_{t 0}^{2}$ while $\Delta A$ changes in proportion to $1 / z_{t 0}$. Thus, DM-KFM is more sensitive to long-range interaction force than CMKFM. If we use an ac bias voltage of $V_{\text {ac }} \cos \left(\omega_{m} t\right) \cot (\omega t)$ instead of $V_{\mathrm{ac}} \cos \left(\omega_{m} t\right) \cos (\omega t)$, we would be able to make $\Delta A$ proportional to $1 / z_{t 0}^{2}$. In that case, however, a highvoltage pulse will be intermittently applied between a tip and a sample, which may influence the sample properties to be measured by KFM.

In this study, we applied the DM method to surface potential measurements by KFM. However, the DM method can be applied to not only KFM but also other surface property measurements such as magnetic force microscopy and photo induced force microscopy. ${ }^{18}$

\section{ACKNOWLEDGMENTS}

This work was supported by a Grant-in-Aid from the Ministry of Education, Culture, Sports, Science and Technology of Japan and the 21st Century Center of Excellence Program, Kyoto University. The authors would like to thank S. Hotta (Kyoto Institute of Technology) for providing M5T molecules.
${ }^{1}$ T. R. Albrecht, D. H. P. Grütter, and D. Ruger, J. Appl. Phys. 69, 668 (1991).

${ }^{2}$ M. Bammerlin, R. Lüthi, E. Meyer, A. Baratoff, J. Lü, M. Guggisberg, C. Gerber, L. Howald, and H.-J. Güntherodt, Probe Microsc. 1, 3 (1997).

${ }^{3}$ F. J. Giessibl, Science 267, 68 (1995).

${ }^{4}$ S. Kitamura and M. Iwatsuki, Jpn. J. Appl. Phys., Part 2 34, L1086 (1995)

${ }^{5}$ S. Kitamura and M. Iwatsuki, Appl. Phys. Lett. 72, 3154 (1998).

${ }^{6}$ B. Gotsmann, C. Seidel, B. Anczykowski, and H. Fuchs, Phys. Rev. B 60, 11051 (1999).

${ }^{7}$ M. Guggisberg, M. Bammerlin, C. Loppacher, O. Pfeiffer, A. A. adn, V. Barwich, R. Bennewitz, A. Baratoff, E. Meyer, and H.-J. Güntherodt, Phys. Rev. B 61, 11151 (2000).

${ }^{8}$ W. Denk and D. W. Pohl, Appl. Phys. Lett. 59, 2171 (1991).

${ }^{9}$ R. Bennewitz, A. S. Foster, L. N. Kantrovich, M. Bammerlin, C. Loppacher, S. Schär, M. Guggisberg, and E. Meyer, Phys. Rev. B 62, 2074 (2000).

${ }^{10}$ C. Loppacher, R. Bennewitz, O. Pfeiffer, M. Guggisberg, M. Bammerlin, S. Schär, V. Barwich, A. Baratoff, and E. Meyer, Phys. Rev. B 62, 13674 (2000).

${ }^{11}$ T. Fukuma, K. Umeda, K. Kobayashi, H. Yamada, and K. Matsushige, Jpn. J. Appl. Phys., Part 1 41, 4903 (2002).

${ }^{12}$ F. J. Giessibl, Noncontact Atomic Force Microscopy (Nanoscience and Technology) (Springer, Berlin, 2002), Chap. 2.

${ }^{13}$ L. Olsson, N. Lin, V. Yakimov, and R. Erlandsson, J. Appl. Phys. 84, 4060 (1998).

${ }^{14}$ K. Okamoto, Y. Sugawara, and S. Morita, Appl. Surf. Sci. 188, 381 (2002)

${ }^{15}$ K. Kobayashi, H. Yamada, H. Itoh, T. Horiuchi, and K. Matsushige, Rev. Sci. Instrum. 72, 4383 (2001).

${ }^{16}$ K. Umeda, K. Kobayashi, K. Ishida, S. Hotta, H. Yamada, and K. Matsushige, Jpn. J. Appl. Phys., Part 1 40, 4381 (2001).

${ }^{17}$ F. J. Giessibl, H. Bielefeldt, S. Hembacher, and J. Mannhart, Appl. Surf. Sci. 140, 352 (1999).

${ }^{18}$ M. Abe, T. Uchihashi, M. Ohta, H. Ueyama, Y. Sugawara, and S. Morita, J. Vac. Sci. Technol. B 15, 1512 (1997). 